\title{
Caffeine in the Treatment of Pain
}

\author{
Cristiane Tavares, TSA ${ }^{1}$, Rioko Kimiko Sakata, TSA ${ }^{2}$
}

\begin{abstract}
Summary: Tavares C, Sakata RK - Caffeine in the Treatment of Pain.
\end{abstract}
Background and objectives: Caffeine is a widely used substance with effects on several systems, presenting characteristic of pharmacokinetic and pharmacodynamic which cause interactions with several drugs. This study's objective is to review the effects caused by caffeine.

Content: This review assesses the caffeine pharmacology, its action mechanisms, indications, contraindications, doses, interactions and adverse effects.

Conclusions: There are insufficient double-blind randomized controlled studies that assess the analgesic effect of caffeine on several painful syndromes. Patients presenting chronic pain need caution when it comes to tolerance development, abstinence and drug interaction from chronic caffeine use.

Keywords: Analgesia; Caffeine; Molecular Mechanisms of Pharmacological Action; Pain.

\section{INTRODUCTION}

Caffeine was isolated in 1820 , but the precise structure of this methylxanthine was established in the last decade of the $19^{\text {th }}$ century. Its effects had not been clearly acknowledged until 1981, when the blockade of adenosine receptors was correlated with caffeine's and its analogues' stimulating proprieties ${ }^{1}$. Caffeine is probably one of the psychoactive substances mostly used in the world, affecting innumerous physiological structures including: physical resistance, humor, sleep and pain ${ }^{2}$. In addition to being consumed as a beverage (coffee and other drinks that contain caffeine), several analgesic drugs - mainly for headaches - contain caffeine associated with paracetamol or with non-hormonal anti-inflammatory drugs ${ }^{3}$. The objective of this study is to review caffeine pharmacology, its action mechanisms and analgesic efficacy in patients presenting chronic pain. This review has been performed using PubMed database and articles published in English since 2000 with caffeine and pain as $\mathrm{MeSH}$ terms.
Received from the Department of Anesthesiology, Pain and Intensive Care, Universidade Federal de São Paulo, Brazil.

1. R4, Pain Sector; Universidade Federal de São Paulo (UNIFESP)

2. PhD; Coordinator at the Pain Sector, UNIFESP

Submitted on February 14, 2011.

Approved on August 3, 2011

Correspondence to:

Rioko Kimiko Sakata, MD

R. Três de Maio 61-51

Vila Clementino

04044020 - São Paulo, SP, Brazil

E-mail: riokoks.dcir@epm.br

\section{Chemical structure}

Caffeine is an alkaloid present in over 60 plant species ${ }^{4}$. Its molecular structure belongs to a trimethyl xanthine group which includes its compounds intimately related: theobromine (present in cacao) and theophylline (present in tea) ${ }^{1}$. These alkaloids are chemically similar to purines, xanthines and uric acid, all metabolically important compounds 4 .

The most common sources of caffeine are: coffee, tea, chocolate and beverages derived from cola ${ }^{1}$. The amount of caffeine present in these beverages varies according to the plant species, coffee bean type, cacao or tea leaf, geographical location, weather, cultural practices and size of the consumed portion ${ }^{4}$.

The amount of caffeine present in a cup of coffee varies between 47 and $134 \mathrm{mg}$. Another important consideration is that coffee, one of the main sources of caffeine, contains hundreds of chemical compounds that may cause or potentiate effects wrongly attributed to caffeine ${ }^{1}$.

In addition to food sources, there are innumerous drugs containing caffeine which in many occasions are sold without prescription ${ }^{4}$.

Caffeine is clinically used as a stimulant of the central nervous system as well as indicated to treat apnea in premature neonates ${ }^{5}$. In this case, it is used as caffeine citrate and administered intravenously. Another formulation is caffeine with sodium benzoate, used as a stimulant or in the treatment of headaches after subarachnoid anesthesia. It is also found in several medications for the treatment of headaches, mostly associated with anti-inflammatory and analgesic drugs ${ }^{3}$.

\section{Pharmacology}

After oral administration, maximum plasma concentration occurs between 30 minutes and 2 hours, which may be prolonged with food intake ${ }^{6}$. Caffeine is completely and read- 
ily absorbed by the intestinal tract, showing bioavailability of $100 \%$ and high solubility both in water and non-polar organic solvents ${ }^{6}$. Its plasma protein binding - mainly albumin - is $10 \%$ to $35 \%{ }^{1}$ and its distribution volume is 0.6 to $0.7{\mathrm{~L} . \mathrm{kg}^{-1}}^{1}$. Caffeine rapidly crosses cell membranes, as well as the blood-brain and placental barrier, reaching great concentrations throughout the body, including the brain ${ }^{7}$.

Twenty-five caffeine metabolites have been described up to date, being metabolized in the liver by CYP1A2 demethylation ${ }^{6}$. The significant differences seen in caffeine plasma concentration in each subject after administration of the same dose are mainly related to variations in metabolisms. Those variations depend on four factors: genetic polymorphisms, cytochrome P450 metabolic induction and inhibition, specific changes (gender and weight), and the presence of hepatic diseases ${ }^{4}$. Cytochrome P450 isoenzyme CYP1A2 subfamily metabolizes most part of caffeine through demethylation $(95 \%)$, transforming it in paraxanthine (85\%), theobromine $(10 \%)$ and theophylline (5\%). Part of the caffeine is metabolized through CYP1A2 in mono-xanthines, which are xanthine oxidase substrate ${ }^{6}$. $\mathrm{N}$-acetyltransferase- 2 metabolizes paraxanthine to AFMU. Other enzymes - such as CYP2E1 and CYP3A3 - also participate albeit to a lesser extent.

Caffeine elimination kinetics is a Michaelis-Menten type, non-linear in high doses, through enzyme saturation ${ }^{1}$. Elimination occurs after caffeine is metabolized into other more polar compounds, such as paraxanthine and theophylline, which also promote biological activity similar to caffeine ${ }^{7}$. Only $1 \%$ to $2 \%$ of the caffeine dose taken is eliminated unchanged in urine ${ }^{1}$. In adults, caffeine elimination half-life is 3 to 5 hours and its metabolites are eliminated in urine. No dose adjustment is required for renal failure ${ }^{6}$. In newborns, both the metabolism and the clearance rate of caffeine are reduced, only achieving the levels found in adults at 6 and 3 months, respectively; its half-life may reach up to 100 hours. In newborns, theophylline metabolism forms caffeine ${ }^{5}$. In smokers, its half-life is twice as high as in non-smokers ${ }^{6}$. In subjects who do not consume coffee, caffeine half-life is twice higher, which explains the higher incidence of intoxication in those patients ${ }^{4}$. Caffeine is secreted in human milk, saliva, bile and semen ${ }^{6}$.

\section{Sites and mechanisms of action}

Caffeine's biological effect seems to be the result of action in several molecular targets ${ }^{8-10}$ :
1. Adenosine receptors
2. Phosphodiesterases
3. Calcium channels
4. $\mathrm{GABA}_{\mathrm{A}}$ receptors
5. Others

Caffeine is a central nervous system stimulant, increasing cyclic 3.5-AMP through the inhibition of phosphodiesterase ${ }^{11}$. Thus, it increases the bulbar respiratory center sensitivity to carbon dioxide, stimulates the central respiratory impulse and increases skeletal muscle contraction, improving diaphragm contractility ${ }^{9}$. Apnea prevention may occur through adenosine competitive inhibition ${ }^{5}$.

\section{Adenosine receptors}

In 1969, adenosine stimulant effects in generating AMPc in brain slices and the blockade of this effect by caffeine were described ${ }^{6,12}$. A few years before, the blockade of adenosine depressing effects in the heart caused by caffeine had been described ${ }^{2}$.

In 1978, Burnstock formulated the existence of two classes of purinergic receptors, named receptors P1 (adenosine) and P2 (ATP). Methylxanthines block P1 receptors and have no effect on P2 receptors. Further studies in the early $80 \mathrm{~s}$ showed that $P 1$ receptors could be divided in $A 1$-adenosine (inhibitors of adenyl cyclase) and A2-adenosine (stimulants of adenyl cyclase) receptors. A2 receptors could be divided in $A 2 a$ (in which agonists show high affinity) and A2b (in which agonists show low affinity) receptors 8,13 .

In 1992, A3 was discovered as a new adenosine receptor in which agonists show low affinity ${ }^{10}$. This receptor, as well as $A 1$, is not only an adenyl cyclase inhibitor, but also a phospholipase-C stimulant, triggering calcium signaling through the genesis of IP3 ${ }^{11}$.

The existence of various adenosine receptor subtypes all sensible to xanthines blockade led to the the development of selective agonists and antagonists ${ }^{1}$.

Adenosine acts in various physiological systems and the effects are often opposed to the ones attributed to caffeine. Thus, most of caffeine pharmacological effects seems to depend on the action of adenosine antagonists on cell surface receptors ${ }^{8,10}$.

Caffeine is a non-selective antagonist of adenosine receptors $\mathrm{A} 1$ and $\mathrm{A} 2 \mathrm{a}$, with an inhibition constant (Ki) of 44 and $40 \mu \mathrm{mol} . \mathrm{L}^{-1}$, respectively. These concentrations correspond well to the plasma concentrations found after a diet with medium amounts of caffeine ${ }^{10}$.

Caffeine acts inhibiting adenosine receptors $\mathrm{A} 1, \mathrm{~A} 2 \mathrm{a}$ and $A 2 b$ and show low affinity for $A 3$ receptors ${ }^{12}$. The antinociceptive effect occurs through the inhibition of $A 2 a$ and $A 2 b$ receptors. But the blockade of $A 1$ receptors that occurs with low doses of caffeine causes the antinociception inhibition of medications such as amitriptyline ${ }^{13}$. This effect on A1 receptors may lead to a decreased analgesic effect of acupuncture and transcutaneous electrical stimulation in patients who consume caffeine either chronically or acutely ${ }^{12}$.

\section{Cyclic phosphodiesterases nucleotide}

There are 3 natural methylxanthines: caffeine, theophylline and theobromine. They are weak competitive inhibitors of phosphodiesterase ${ }^{1}$. 
Caffeine inhibits phosphodiesterase, increasing the effect and the action duration of intracellular AMPc ${ }^{4}$. Therefore, the effects of catecholamines are potentiated. Methylxanthines also increase the release of catecholamines ${ }^{14}$. The inhibition constant for this effect (phosphodiesterase inhibition) is $480 \mu \mathrm{mol} . \mathrm{L}^{-1} 10$.

\section{Calcium release}

Caffeine activates the calcium channels sensible to ryanodine found in the endoplasmic and sarcoplasmic reticulum, leading to the release of intracellular calcium. Millimolar concentrations of caffeine, required to activate the channels, ${ }^{11}$ also trigger other effects on calcium homeostasis such as the inhibition of channels sensible to IP3 1 .

Up to date, caffeine seems to be the most potent and selective xanthine for calcium channels sensible to ryanodine ${ }^{15}$.

This effect, as well as the phosphodiesterase inhibition effect, does not seem to occur in vivo in regular conditions, since a much higher concentration than the caffeine therapeutic concentration (100 to $1,000 \mu \mathrm{mol} . \mathrm{L}^{-1}$ ) is required for these effects to take place, which acquire great importance in an intoxication setting ${ }^{10}$.

\section{$G A B A_{A}$ receptors}

Caffeine interacts with $\mathrm{GABA}_{A}$ receptors ${ }^{1}$. Caffeine and theophylline act as reverse antagonists or agonists in benzodiazepines' action sites ${ }^{11}$; that is, they act by blocking GABA $_{A}$ receptors ${ }^{12}$.

However, the required caffeine concentrations to promote this effect are hundreds of times higher than the caffeine concentrations achieved with a regular diet ${ }^{12}$.

\section{Other action sites}

There are other potential sites of caffeine action, but millimolar concentrations of this substance are generally required. There are several ionic channels, with neurotransmitters release and action in several enzymes ${ }^{9}$. The effect on enzymes and ionic channels is generally inhibitory. However, Na/K-ATPase stimulation by caffeine and theophylline occurs in millimolar concentrations 6,8 . The activation of calcium entrance and potassium and calcium channels was also reported to have been caused by caffeine. Caffeine also seems to sensitize Mg-ATPase to the stimulating effects of calcium in the cardiac myofibrils ${ }^{1}$.

\section{Effects}

\section{Renal}

Caffeine and theophylline diuretic and natriuretic effects are well known and both have been used to treat congestive heart failure associated edema. The diuretic effect occurs through antagonists of adenosine $\mathrm{A} 1$ and $\mathrm{A} 2 \mathrm{a}$ receptors ${ }^{8}$.

\section{Respiratory}

Theobromine antitussive effects are well known. Caffeine is widely used to treat apnea occurring in premature newborns, with a minimum incidence of adverse effects 5 .

The xanthines' stimulating respiratory effects occur due to adenosine receptor antagonism in respiratory centers. Caffeine increases sensibility of the respiratory center to carbon gas ${ }^{6}$. However, peripherally, caffeine has an inhibitory effect on respiration through the blockade of adenosine A2 receptors in the carotid body ${ }^{8}$.

Caffeine seems to improve pulmonary function in asthmatic patients, probably due to it increasing the effect of bronchodilators ${ }^{1}$.

\section{Other effects}

Caffeine produces antidepressant, anxiolytic, neuroprotector effects and improves cognitive function ${ }^{2}$. The blockade of A2a receptors may be of value in preventing hepatic cirrhosis.

In people who do not consume coffee, caffeine half-life is twice as high, which explains a higher incidence of intoxication in this group ${ }^{4}$.

\section{Indications}

Caffeine citrate is exclusively used for the apnea treatment in premature neonates ${ }^{5}$.

Caffeine is used for the respiratory depression treatment in adults, with intramuscular or intravenous administration ${ }^{1}$.

Xanthines and its heterocyclic analogues have been developed for Alzheimer's disease treatment ${ }^{16}$. Consuming beverages that contain caffeine seems to prevent this disease ${ }^{17}$. Studies show that in cortical neurons, intracellular calcium release is significantly higher in rats that received caffeine 18,19 .

There is extensive literature regarding possible anticancer effects of caffeine and other xanthines ${ }^{1,2}$. Caffeine's antimetastatic effect has been reported in a rat breast tumor model 20 .

Antagonists of adenosine A2b receptors have been suggested for the treatment of type 2 diabetes mellitus, but the attempts to correlate the caffeine use to the risk of this disease were inconsistent ${ }^{11}$. However, there have been reports that the intake of caffeine causes reduction in the uptake of insulin-stimulated glucose ${ }^{21}$.

Antagonists of adenosine A2a receptors seem to have a role in the Parkinson's disease treatment ${ }^{17}$, and the use of beverages containing caffeine seems to be associated with a lower risk to develop the disease ${ }^{18}$.

\section{Caffeine for the treatment of pain}

Caffeine has a long history as an analgesic drug adjuvant ${ }^{6}$. Other adenosine receptor antagonists have antinociceptive effects, and the A2b receptors seem to be the target ${ }^{22}$. 
In a review, authors show preclinical studies of caffeine intrinsic antinociceptive action ${ }^{4}$. The antinociceptive dose ranged between 25 and 100 mg. $\mathrm{kg}^{-1} 12$.

The antinociception seems to occur mainly through the blockade of adenosine $\mathrm{A} 2 \mathrm{a}$ and $\mathrm{A} 2 \mathrm{~b}$ receptors ${ }^{22}$. Other mechanisms not related to the blockade of adenosine, such as changes in the activity and synthesis of cyclooxygenase enzymes in certain sites are also implied in the adjuvant analgesic effect of caffeine ${ }^{12}$.

\section{Controlled studies}

There are few controlled randomized double-blind studies that assess caffeine analgesic efficacy ${ }^{6}$.

In one study, there has been improvement of the cognitive function in oncologic patients treated chronically with morphine and who received a morphine dose plus $200 \mathrm{mg}$ of caffeine in bolus intravenously. A decrease in pain intensity was observed in the group of patients who received caffeine with morphine, but with no statistical difference regarding the group that received morphine with placebo ${ }^{23}$.

In a meta-analysis authors suggest that due to the caffeine vasoconstrictor effect caused by the adenosine receptors blockade, it may be useful for headache relief ${ }^{12}$. In a doubleblind controlled prospective study in patients with acute onsets of migraine without aura, the use of $100 \mathrm{mg}$ of caffeine associated with oral $100 \mathrm{mg}$ of sodium diclophenac showed that the analgesic effect was superior to diclophenac alone or to placebo in the first hour after administration, without significant adverse effects ${ }^{24}$.

In a clinical trial in patients with tension headache, $301 \mathrm{pa}-$ tients were divided in four groups: first group patients received ibuprophen $400 \mathrm{mg}$ associated to caffeine $200 \mathrm{mg}$; second group, ibuprophen $400 \mathrm{mg}$; third group, caffeine $200 \mathrm{mg}$; and fourth group, placebo. Patients in the first group showed a faster and more significant improvement of headache. The ones who received caffeine alone showed a similar improvement to those who received ibuprophen alone, and both groups showing significant difference in relation to the ones who received placebo. In addition, patients who received caffeine showed faster relief of pain than those who received ibuprophen alone. However, the ones who received caffeine reported more adverse effects - mainly nervousness, nausea and dizziness ${ }^{25}$.

Abrupt interruption in patients who make chronic use of caffeine (as in preoperative fasting, for instance) may cause abstinence syndrome starting at 12 to 24 hours, peaking at 24 to 48 hours, and lasting up to 1 week. Main symptoms are headache and fatigue, which show improvement with caffeine oral or intravenous administration ${ }^{12}$.

On the other hand, the regular use of caffeine may be associated to the development of migraines and daily chronic headaches mainly in children ${ }^{12}$.

Regarding caffeine adjuvant analgesic effect in other conditions, results are very poorly consistent, but as noted before, there are few randomized prospective controlled trials available 6,12 .
In postoperative pain, caffeine association seems to poorly increase the analgesic effect of paracetamol, and to provide no increase at all of ibuprophen ${ }^{12}$. In another study, caffeine has showed to increase and prolong the analgesic effect of paracetamol ${ }^{26}$. The authors have conducted a pharmacokinetic study and observed that caffeine accelerates paracetamol absorption. This can be explained by the blood flow increase in the gastrointestinal mucosa. In addition, both caffeine and paracetamol are cytochrome P450 substrates, and caffeine is a weak inhibitor of this enzyme.

In another study, using an ischemic pain model, authors did not obtain any analgesic effect with $10 \mathrm{mg} \cdot \mathrm{kg}^{-1}$ of intravenous caffeine. In this study, 17 health volunteers were assessed, who received infused caffeine or placebo with inflated tourniquet in the non-dominant arm and were required to lift up a $3 \mathrm{~kg}$-weight several times until they complained of unbearable pain or until 45 minutes had passed ${ }^{27}$.

Regarding headaches after subarachnoid anesthesia, there is no double-blind randomized controlled study that proved caffeine to be effective ${ }^{28}$. In spite of that, it is widely used for such purpose at doses ranging between 300 and $500 \mathrm{mg}^{12}$.

\section{Administration modes}

Caffeine may be orally administered regardless of food intake.

Caffeine citrate for injection may be orally administered ${ }^{6}$. Parenterally, caffeine citrate loading dose may be infused in at least 30 minutes. Maintaining dose must be administered in at least 10 minutes. It may be administered without being diluted or diluted in glucose serum in a concentration of $10 \mathrm{mg} \cdot \mathrm{mL}^{-1} 4$. Caffeine with sodium benzoate may be administered via intramuscular with dilution, intravenous slowly, or diluted in physiologic serum ${ }^{6}$.

\section{Caution}

Caffeine should be used with caution in patients with peptic ulcer disease, gastroesophageal reflux disease, renal or hepatic function impairment or cardiovascular disease. Its use must be avoided in patients with symptomatic cardiac arrhythmias, agitation, anxiety and tremors ${ }^{4}$.

Caffeine dose present in drugs ranges from 30 to $100 \mathrm{mg}$. In these cases, one should pay attention to the concomitant use of beverages and foods containing caffeine ${ }^{7}$.

Caffeine associated with sodium benzoate should not be switched to the caffeine citrate formulation ${ }^{6}$.

The use of sodium benzoate in neonates should be avoided as it has been associated with asphyxia syndrome. Neonates who receive caffeine citrate must be closely monitored as there is risk of necrotizing enterocolitis ${ }^{5}$.

Risk factor for pregnancy is level C. Caffeine crosses the placenta, and plasma concentration in the fetus are similar to those in the mother. There are reports of teratogenic ef- 
fects with the administration of high in bolus doses in animals. Moderate caffeine consumption is not associated to congenital malformations, spontaneous miscarriage, prematurity or low birth weight. Pregnant women who do not smoke or drink alcohol may consume up to $5 \mathrm{mg} \cdot \mathrm{kg} \cdot \mathrm{d}^{-1}$ according to some sources, but others limit such use to $150 \mathrm{mg}^{\mathrm{d}} \mathrm{d}^{-1}$. Caffeine half-life is prolonged during the second and third semesters of pregnancy 22,29 .

Some studies in animals showed that caffeine's possible effects when used during pregnancy are skeletal alterations, intrauterine growth retardation and low birth weight ${ }^{6}$. The use of a large amount of caffeine seems to be associated with miscarriage and congenital malformations in humans ${ }^{22}$.

Caffeine has shown to be present in human milk ${ }^{6}$.

\section{Contraindications}

It is contraindicated in cases of hypersensitivity to caffeine or to any component of the formulation ${ }^{22}$.

\section{Pharmacological interactions}

Caffeine concentration may be reduced with the induction of its metabolism. Among the inductors are the use of tobacco, carbonized meat, some vegetables, low body mass index, male subjects, and regular coffee use itself, as well as the use of rifampicin, benzodiazepines, carbamazepine, phenobarbital drugs and omeprazole. Tobacco induces caffeine metabolism and, as a result, reduces its plasma concentrations ${ }^{6}$. Smokers who consume coffee and quit smoking may experience caffeine intoxication symptoms as caffeine doubles its concentration in the absence of tobacco.

Caffeine concentrations may increase with the inhibition of its metabolism. This occurs in the end of pregnancy, in female patients with liver disease and obesity, in some food and alcohol intake and with the use of some medications such as antimycotic drugs (fluconazole, ketoconazole), antiarrhythmic drugs (diltiazem, verapamil), antidepressants (paroxetine, fluoxetine, fluvoxamine), antipsychotic drugs (clozapine, olanzapine), methylxanthines (theophylline), oral contraceptive, cimetidine, quinolones and allopurinol 4,6 .

Some drugs cause an increase in caffeine effect and toxicity: quinolones (particularly ciprofloxacin and ofloxacin) and CYP1A2 inhibitors such as ketoconazole and fluvoxamine ${ }^{2}$.

Caffeine may reduce sedative and anxiolytic effects of benzodiazepines and barbiturates ${ }^{2}$. It increases paracetamol, acetylsalicylic acid and ergotamine absorption and bioavailability. It reduces the clearance of theophylline and competitively inhibits clozapine metabolism, which may increase their plasma concentrations and likelihood of adverse effects 4 .

Caffeine produces additive analgesic effect when simultaneously administered, particularly with non-hormonal antiinflammatory drugs. In association with paroxetine, it may cause serotonin syndrome. In addition, it potentiates nicotine stimulant effects and intensifies alcohol, nicotine and vasoconstrictors teratogenic effects ${ }^{4,6}$.
In some experimental models ${ }^{12}$, caffeine in low doses seems to inhibit several agents' antinociceptive effects such as amitriptyline, venlafaxine, carbamazepine and paracetamol. This seems to be related to the blockade of adenosine type A1 receptors, which present antinociceptive effect ${ }^{13}$. This effect also seems to be important in analgesia related to acupuncture and TENS, which theoretically could be inhibited by the use of caffeine ${ }^{12}$.

\section{Acute adverse reactions}

Due to significant variability among subjects, the same dose of caffeine may cause adverse reactions in one person and show good tolerability in another. Most frequent adverse effects are palpitation, tachycardia, gastric changes, tremor, nervousness and insomnia ${ }^{7}$.

High doses may cause intense anxiety, fear and anguish crisis ${ }^{6,7}$. Cases of acute caffeine-induced psychosis in patients showing no psychopathology and worsening of psychotic symptoms in schizophrenic patients are described ${ }^{16}$. Both coffee and tea may form insoluble compounds when associated with psychiatric medications, reducing its efficacy when administered concomitantly 4 .

Caffeine may cause anaphylaxis and be an allergen that causes unknown urticaria among consumers of cola-derived beverages ${ }^{6}$.

Cardiovascular (angina, arrhythmia, chest pain, palpitation, flushing and vasodilatation), gastrointestinal (gastritis, reduction of esophageal sphincter tonus), neuromuscular skeletal (fasciculation), eye disorders (intraocular pressure increase with caffeine dose $>180 \mathrm{mg}$ and miosis), renal (diuresis increase) effects and effects in the central nervous system (agitation, hallucinations, headache, delirium, restlessness, insomnia, irritability, dizziness and psychosis) ${ }^{8}$ are also possible.

\section{Undesirable effects of chronic exposure}

There is no clear evidence that the exaggerated use of caffeine causes a significant risk to the health of healthy subjects. Caffeine intake is not directly related to the risk of hypertension. Despite altering lipid metabolism and endothelial function, there are contradictory reports on whether or not there is an increased risk of coronary disease ${ }^{1,2}$.

Caffeine reduces bone density as it may potentiate the action of glucocorticoids. It does not prevent nor limits the gain of weight in overweight patients ${ }^{2}$.

There is no indication that caffeine produces nephropathy alone neither does it increase the incidence of nephropathy caused by analgesic drugs. Caffeine increases calcium excretion in the urine and is one of the multiple factors implied in female urinary incontinence. Caffeine increases sodium urinary excretion and has a diuretic effect, with intensity reduced in chronic users ${ }^{6}$. 
Caffeine stimulates the secretion of gastric acid and colon activity. The association of the use of caffeine with the development of gastric or duodenal ulcers is not clear. However, the exaggerated use of caffeine exacerbates gastroesophageal reflux, but it is not known whether this effect is caused by caffeine or other substances contained in coffee apart from caffeine ${ }^{2,6}$.

The use of caffeine is associated to conception delay, increase of spontaneous miscarriages rates and reduction of fetal growth ${ }^{6}$.

There is not enough information regarding long-term adverse effects of caffeine use in preterm neonates. In the child and adolescent population, it may cause chronic daily headaches and migraines without aura, which disappears when coffee intake is reduced or stopped ${ }^{22}$.

In some subjects, caffeine causes pathologic sleep because it produces paradoxical sedation, an idiosyncratic phenomenon which has also been described with amphetamines. Caffeine abstinence causes hypersomnia ${ }^{6}$.

Caffeine may cause anxiety, anguish crisis, abstinence, and sleep dependence and alterations ${ }^{2,6}$.

Caffeine may change the cellular cycle control functions and many mechanisms of DNA reparation, as well as increase the antagonism to exposure of mutagenic and carcinogenic potentials. Even if the results are contradictory, a correlation between pancreas cancer and the use of caffeine has been suggested. Gene K-ras mutation, the tumor marker for exocrine pancreatic cancer, increases in a dose-dependent manner with the use of coffee ${ }^{20}$.

On the other hand, several studies have related the chronic use of coffee with the risk reduction for colorretal cancer, but no prospective study confirmed this result. In addition to that, caffeine shows suppressing effects on tumor cells in experimental metastasis ${ }^{1,20}$.

\section{Intoxication}

Most frequent intoxications occur in people who consume coffee, but that happens with users who increase their regular dose or with regular consumers of caffeine high doses. Most frequent symptoms are tachycardia, restlessness, nervousness, tremor and insomnia ${ }^{\text {? }}$.

Caffeine intoxication, even in a large amount, may not show due to tolerance development ${ }^{4}$.

Other manifestations are: comma with pulmonary edema, arrhythmias (from sinus tachycardia to atrial or ventricular fibrillation), myocardial infarction and rhabdomyolysis 1,4 .

The estimated acute lethal dose in adults is 5 to $10 \mathrm{~g}$, intravenously or orally. Caffeine intoxication lethal cases are described ${ }^{30}$ and there is a report of a case of a patient who survived $24 \mathrm{~g}$ of caffeine ${ }^{31}$.

Caffeine chronic toxicity may be manifested as myopathy, hypokalemia, muscle weakness, nausea, vomiting and diarrhea and weight reduction ${ }^{30}$.

\section{Caffeine as a drug of abuse}

Caffeine classification as a drug of abuse is a polemic subject. If the definition by DSM-IV-TR (Diagnostic and Statistical Manual of Mental Disorders, $4^{\text {th }}$ edition, Text Revision) for dependence is taken into account, seven criteria must match to classify a patient as caffeine dependent. The majority of caffeine consumers reach at least three criteria: use caffeine in greater amounts or for a larger period than the what was initially intended, tolerance development and abstinence symptoms ${ }^{2,7}$.

Moderate consumers are the ones who consume 128 to $595 \mathrm{mg} \cdot \mathrm{dia}^{-1}$. Major consumers use 1,020 to $1,035 \mathrm{mg}^{-\mathrm{dia}^{-1}}{ }^{17}$.

Tolerance is defined as the need to consume an increasingly higher dose of certain substance to obtain the same effect that was obtained with the initial dose. Tolerance to caffeine is a phenomenon of quick onset, low magnitude and crossed with the ones showed with other methylxanthines, but with a distinct mechanism from other drugs such as amphetamine and methylphenidate ${ }^{7}$.

The tolerance phenomenon could be justified by the nonlinear accumulation of caffeine and its main metabolites in multiple-dose models ${ }^{6}$.

In humans, tolerance to effects on blood pressure, heart rate, diuresis, adrenaline and noradrenaline plasma levels and renin activity, as well as on sleep alterations has been demonstrated to occur within a few days ${ }^{7}$.

\section{Behavior effects}

The use of coffee and beverages that contain coffee by millions of people in the world is linked to the stimulating proprieties of caffeine. Caffeine increases alertness sensation, fights fatigue and generally provides a sensation of well-being ${ }^{2}$.

Addiction does not seem to occur, although abstinence symptoms seem to be present ${ }^{7}$. In high doses and in certain subjects, uncomfortable effects may occur, such as anxiety.

The molecular targets responsible for caffeine behavior effects have been extensively investigated primarily in rodents. They are mainly caused by the blockade of adenosine receptors, but the relative role of each subtype is still under investigation. The dopaminergic system seems to be involved ${ }^{16}$.

It is important to emphasize that caffeine's acute and chronic effects may be quite varied.

The majority of caffeine's central effects in concentrations found in beverages is due to the blockade of adenosine receptors ${ }^{8}$.

Anxiolytic effects of a xanthine with an extended ring that contains an arylpiperazine component seem to be due to the agonist activity on serotoninergic receptors ${ }^{4}$.

\section{Genetic aspects}

The existence of a common genetic factor in the consumers of drugs acting as adjuvants, coffee use or abuse together with alcohol or tobacco, has been demonstrated. There is polymorphism for gene CYP1A2 of slow caffeine metabolizers which show greater risk for myocardial infarction ${ }^{6}$. 


\section{Abstinence syndrome}

The onset of the abstinence symptoms ranges from 12 to 24 hours after caffeine use interruption, with maximum intensity ranging from 24 to 48 hours and duration of 2 to 9 days. Headache is the most frequent symptom ${ }^{7}$. Tiredness, fatigue, reduction of short and long-term alertness state also occur. In a minor level, depressing humor, difficulty concentrating, irritability and de-motivation during work may occur as well. Most severe manifestations of the abstinence syndrome are tachycardia, shaking hands, reduction of blood pressure, motor activity and adrenaline excretion in urine ${ }^{3}$.

\section{CONCLUSION}

Caffeine is a widely consumed substance both through diet habits and medications that in many occasions are sold without a doctor's prescription. It shows many interactions with several medications and effects in all systems, with differences regarding acute and chronic use. It may cause dependence and abstinence syndrome.

Regarding pain treatment, there are few randomized prospective controlled studies, but they seem to show an analgesic efficacy in the treatment of tension headache and migraine without aura, in addition to showing an increased and prolonged analgesic effect of paracetamol through pharmacodynamic interaction. Its use in patients with chronic pain is limited by the incidence of side effects, abstinence and the risk for dependence, although it may be useful to improve the cognitive performance in cancer patients using morphine. Regarding headaches after subarachnoid anesthesia, there are no consistent studies that prove its efficacy. 


\section{REFERÊNCIAS/REFERENCES}

1. Daly JW - Caffeine analogs: biomedical impact. Cell Mol Life Sci, 2007;64:2153-2169.

2. Fredholm BB, Battig K, Holmen $\mathrm{J}$ et al. - Actions of caffeine in the brain with special reference to factors that contribute to its widespread use. Pharmacol Rev, 1999;51:83-133.

3. Goldstein J, Silberstein SD, Saper JR et al. - Acetaminophen, aspirin and caffeine in combination versus ibuprofen for acute migraine: results from a multicenter, double- blind, randomized, parallel-group, single-dose, placebo- controlled study. Headache, 2006;46:444-453.

4. Sawynok J - Methylxanthines and pain. Handb Exp Pharmacol, 2011;200:311-329.

5. Schmidt B, Roberts RS, Davis $P$ et al. - Caffeine therapy for apnea of prematurity. N Engl J Med, 2006;354:2112-2121.

6. Sawynok J, Yaksh TL - Caffeine as an analgesic adjuvant: a review of pharmacology and mechanisms of action. Pharmacol Rev, 1993;45:43-85.

07. Juliano LM, Griffiths RR - A critical review of caffeine withdrawal: empirical validation of symptoms and signs, incidence, severity, and associated features. Psychopharmacology, 2004;176:1-29.

8. Ribeiro JA, Sebastião AM - Caffeine and adenosine. J Alzh Dis, 2010;20:S3-S15.

9. Davis JK, Green JM - Caffeine and anaerobic performance: ergogenic value and mechanisms of action. Sports Med, 2009;39(10):813832.

10. Carrillo JA, Benitez $\mathrm{J}$ - Clinically significant pharmacokinetic interactions between dietary caffeine and medications. Clin Pharmacokinet, 2000;39(2):127-153.

11. Shi D, Padgett WL, Daly JW - Caffeine analogs: effects on ryanodinesensitive calcium-release channels and GABAa receptors. Cell Mol Neurobiol, 2003;23:331-347.

12. Sawynok J - Caffeine and pain. Pain, 2010; doi:10.1016 (in press).

13. Sawynok J, Reid AR, Fredholm BB - Caffeine reverses antinociception by amitriptyline in wild type mice but not in those lacking adenosine A1 receptors. Neurosci Lett, 2008;440(2):181-184.

14. Castro A, Jerez MJ, Gil C et al. - Cyclic nucleotide phosphodiesterases and their role in immunomodulatory responses: advances in the development of specific phosphodiesterase inhibitors. Med Res Rev, 2005;25:229-224. 
15. Cheng LZ, Lü N, Zhang YQ et al. - Ryanodine receptors contribute to the induction of nociceptive input-evoked long-term potentiation in the rat spinal cord slice. Mol Pain, 2010;20:1-6.

16. Cauli $\mathrm{O}$, Morelli $\mathrm{M}-$ Caffeine and the dopaminergic system. Behav Pharmacol, 2005;16:63-77.

17. Xu K, Bastia E, Schwarschild M - Therapeutic potential of adenosine A2a receptor antagonists in Parkinson's disease. Drugs Aging, 2005;22:471-482.

18. Maia $L$, De Mendonça A - Does caffeine intake protect from Alzheimer disease? Eur J Neurol, 2002;9:377-382.

19. Arendash GW, Schleif W, Rezai-Zadeh K et al. - Caffeine protects Alzheimer's mice against cognitive impairment and reduces brain betaamyloid production. Neuroscience, 2006;142:941-952.

20. Yang $H$, Rouse J, Lukes $L$ et al. - Caffeine suppresses metastasis in a transgenic mouse model: a prototype molecule for prophylaxis of metastasis. Clin Exp Metastasis, 2004;21:719-735.

21. Petrie HJ, Chown SE, Belfie LM et al. - Caffeine ingestion increases the insulin response to na oral-glucose- tolerance test in obese men before and after weight loss- Am J Clin Nutr, 2004;80:22-28.

22. Zhang $W Y-A$ benefit- risk assessment of caffeine as an analgesic adjuvant. Drug Safety, 2001;24:1127-1142.

23. Mercadante S, Serretta R, Casuccio A - Effects of Caffeine as an adjuvant to morphine in advanced cancer patients: a randomized, double-blind, placebo-controlled, crossover study. J Pain Symptom Manage, 2001;21(5):369-372.

24. Peroutka S, Lyon JA, Swarbrick J et al. - Efficacy of diclofenac sodium softgel $100 \mathrm{mg}$ with or without caffeine $100 \mathrm{mg}$ in migraine without aura: a randomized, double-blind, crossover study. Headache, 2004;44:136-141.

25. Diamond S, Balm TK, Freitag FG - Ibuprofen plus caffeine in the treatment of tension-type headache. Clin Pharmacol Ther, 2000;68(3):312319.

26. Renner B, Clarke G, Grattan T et al. - Caffeine accelerates absorption and enhances the analgesic effect of acetaminophen. J Clin Pharmacol, 2007;47:715-726.

27. Ewans RW, Armon C, Frohman EM et al. - Assessment: prevention of post-lumbar puncture headaches. Neurology, 2000;55:909-914.
28. Lin W, Geiderman J - Myth: fluids, bed rest, and caffeine are effective in preventing and treating patients with post-lumbar puncture headache. West J Med, 2002;176(1):69-70.

29. Palmer $H$, Graham G, Williams $K$ et al. - A risk-benefit assessment of paracetamol (acetaminophen) combined with caffeine. Pain Med, 2010;11(6):951-965.

30. Feinstein AR, Heinemann LA, Dalessio D et al. - Do caffeine-containing analgesics promote dependence? A review and evaluation. Clin Pharmacol Ther, 2000;68(5):457-467.

31. Morelli M, Simola N - Methylxantines and drug dependence: a focus on interactions with substances of abuse. Handb Exp Pharmacol, 2011;200:483-507. 